\title{
Boundary and size estimation of debonds in external wall finishes of high-rise buildings using Infrared thermography
}

by W.L. Lai and C.S. Poon

* Dept. of Civil and Structural Engineering, The Hong Kong Polytechnic University, Hong Kong.

celaiwl@inet.polyu.edu.hk, cecspoon@inet.polyu.edu.hk

\begin{abstract}
This paper reports an infrared thermography (IRT) application on boundary and size estimation of debonds in rendered composites in high rise residential buildings. The application comprises the use of the traditional passive infrared thermography method, digitizing the infrared images to linear gray-scale, and processing the pixel array using a simple spatial pixel differentiation algorithm. The validity of the methodology was verified by comparing the estimation with the actual examination of the debonds and the error was found to be less than $16 \%$.
\end{abstract}

\section{Introduction}

In Asia's densely populated cities, debond evaluations of tile-adhesive and render composites in external finishes of tall residential buildings $(30+$ storeys) are often based on three traditional methods: visual inspection, hammer-tapping and pull-off tests. Though none of these tests are effective in a large-scale inspection, they are still customary used for preparation of rehabilitation scheme and life-cycle cost analysis in Hong Kong. But these methods do not provide early warning before bond failures and do not comply the technical aspect specified in the recent mandatory building inspection scheme (MBIS) stipulated by the Hong Kong government. The drawbacks of the above three methods can be circumvented by infrared thermography (IRT). Embedded debonds can be detected non-destructively, remotely and effectively by using non-contact and nondestructive IRT. The underlying principle of this assessment technique is that the entrapped air in the debonded area makes its surface temperature hotter than the areas without debonds after a period of exposure to natural sunlight. So when a non-uniform temperature distribution is observed over an external wall surface, it may indicate the presence of debonds, provided that it cannot be explained due to other reasons such as structural detailing, effects of other heat sources, variations of emissivity of the concerned materials etc [1-4]. Despite the IRT is available to identify debond qualitatively since the last decade, and has been widely adopted in Hong Kong and other densely populated Asia cities, a quantitative method that can estimate the boundaries and sizes of the debond is still lacking. A quantitative method is needed to serve as a screening tool before subsequent pull-off or hammer tapping tests are performed. It is because boundary estimation of debonds using IRT requires sophisticated programming skills and in-depth understanding of transient heat transfer which most practitioners are not familiar with.

A major disadvantage of the qualitative IRT assessment is it is based on an artificially adjusted temperature range for capturing the thermogram. The decision of the choice of the temperature adjustment range relies purely on the experience of the operator and does not follow any guideline established by scientific principles. It often leads to misleading interpretations such as a larger or a smaller area of debond, or even no debond is present. An example shown in Fig. 1 illustrates four different thermograms derived from the same set of raw data, in which the chosen temperature ranges were from $22-34^{\circ} \mathrm{C}, 22-36^{\circ} \mathrm{C}, 28-40^{\circ} \mathrm{C}, 22-48^{\circ} \mathrm{C}$. The four thermograms show different extent of temperature's variation over the same debond area, although they are based on the same set of raw temperature data. At the moment, this arbitrary adjustment by the operator is not standardised by any objective guideline. As a result, the actual size of the debond cannot be estimated accurately. Therefore, we propose a systematic image processing method to carry out the quantitative assessment. The method is based on processing the converted gray-scale images with spatial pixel differentiation methods developed by the authors previously [5-7]. A clear advantage is that as soon as a debond can be identified qualitatively, its size and boundary are estimated irrespective of the chosen temperature range. The accuracy of this method is studied with variations in three categories of factors (environmental, materials and instrumentation). The environmental factor refers to changes of heat flux intensity, temperature differentials between the surface render and the ambient environment, direct or oblique exposure to the sun and wind speeds. The materials factor includes the heat transfer properties and emissivity of the concerned materials, and the instrumentation factor studies the effect of different illumination angles of the IR thermo-imager to the Sun and high- or low-end types of IR thermo-imager on the results. In this paper, only effects of the variation of the the heat flux intensities and temperature differentials between the surface render and the ambient environment are reported. 


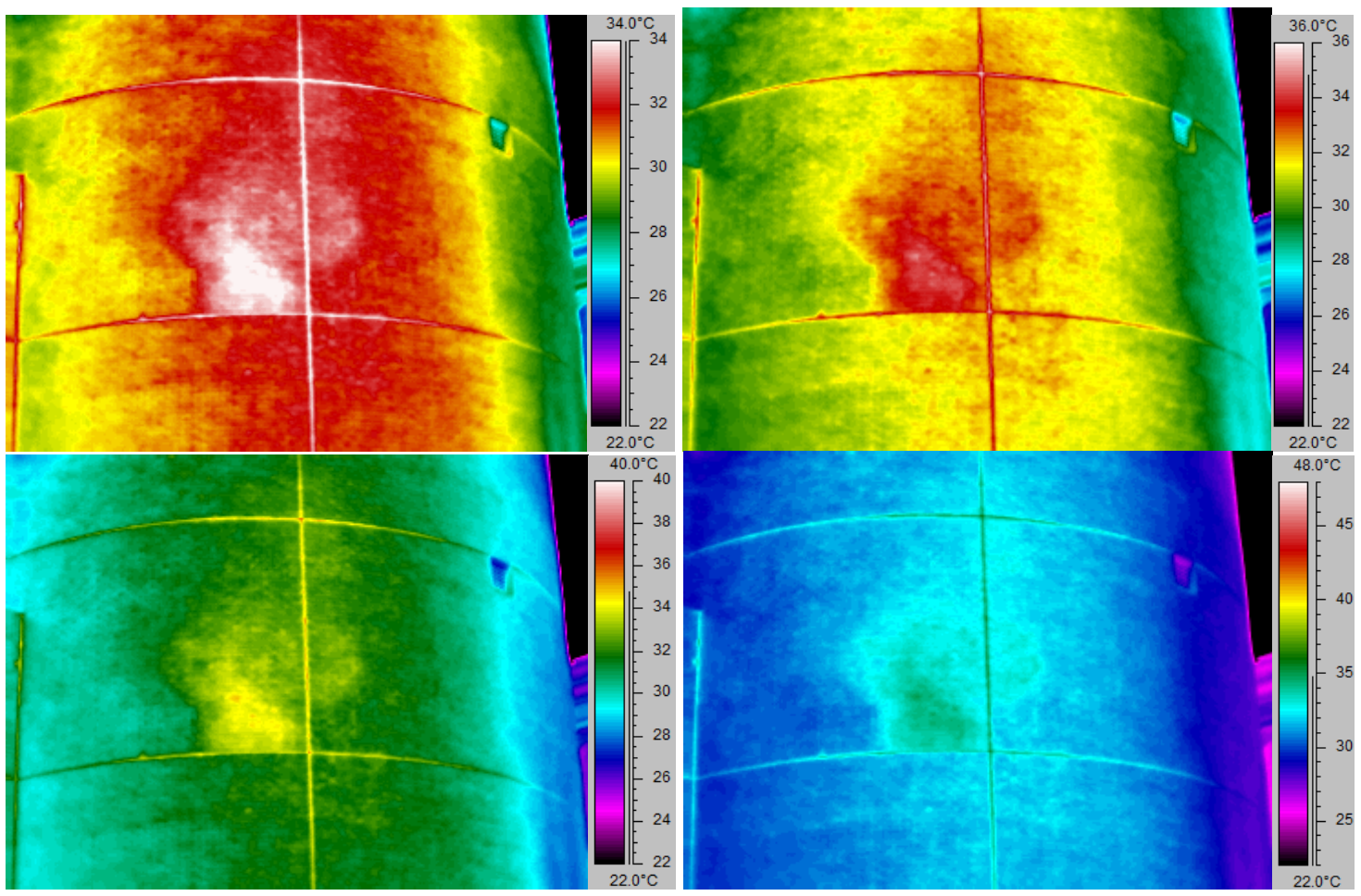

Fig.1 Effects of adjusted temperature ranges and mid-temperature on the shape of debond

\section{Materials}

Four concrete panels each with size $600 \mathrm{~mm}(\mathrm{~L}) \times 290 \mathrm{~mm}(\mathrm{~W}) \times 100 \mathrm{~mm}(\mathrm{~T})$ were prepared in the laboratory and a proprietary base plaster (Optimix, BP126) [8] was applied on each of the panel. In each panel, nine circular plastic foam plates $(7.5 \mathrm{~cm}$ diameter) with seven different thickness were embedded in the $23 \mathrm{~mm}$ thick render layer (Fig. 2 diagram ' $a$ ' and ' $b$ '). The panels were put on a roof of a building and oriented to face east so that they were subjected to direct sunlight in the morning.

\section{Measurement methodology}

The measurement was divided into two parts: (1) qualitative detection and (2) size and boundary quantification. The first part was based on the continuous measurement of the abnormal heat distribution over the specimen surfaces using a FLIR quantum well SC3000 infrared thermo-imager (Fig. 2, diagram ' $a$ ' and 'b'). The temperature date was captured once every minute, via instrumentation controlled by a National Instrument's hardware and a program made in LabVIEW software. A heat flux plate and three K-type thermocouples were also installed on the surface of the top right panel (Fig. 2 diagram 'a') to monitor the heat flux and temperature and data was taken once every minute. Another thermocouple was also used to measure the ambient temperature. The natural heating cycle was : firstly absorption of heat energy in the morning, and secondly release of heat energy in the afternoon. The paper focuses on the heat absorption part to the cycle, while the results of the latter heating cycle (heat release) are out of the scope of this paper.

The digitized gray scale infrared images taken (Fig. 2, diagram ' $c$ ' and 'd'), were processed with spatial pixel differentiation methods (Fig. 2, diagram 'e') developed which were based on our previous work on studying of CFRPconcrete composites [5-7,9]. The nine debond areas can be identified clearly by the infrared thermo-imager (Fig. 2 diagram 'b'). The arrays of image pixel were then processed to define the steepest temperature gradient computed over the debonds [1,10]. Such computation may be processed with a number of algorithms, such as Roberts gradient [11], high degree approximation [1], second order fit [1], inflection point [5-7,12]. It should be noted that using this method, as long as the debond is visible, the temperature range chosen in the thermograms was no longer important. This has overcome the limitations discussed in Section 1 and Fig. 1. 


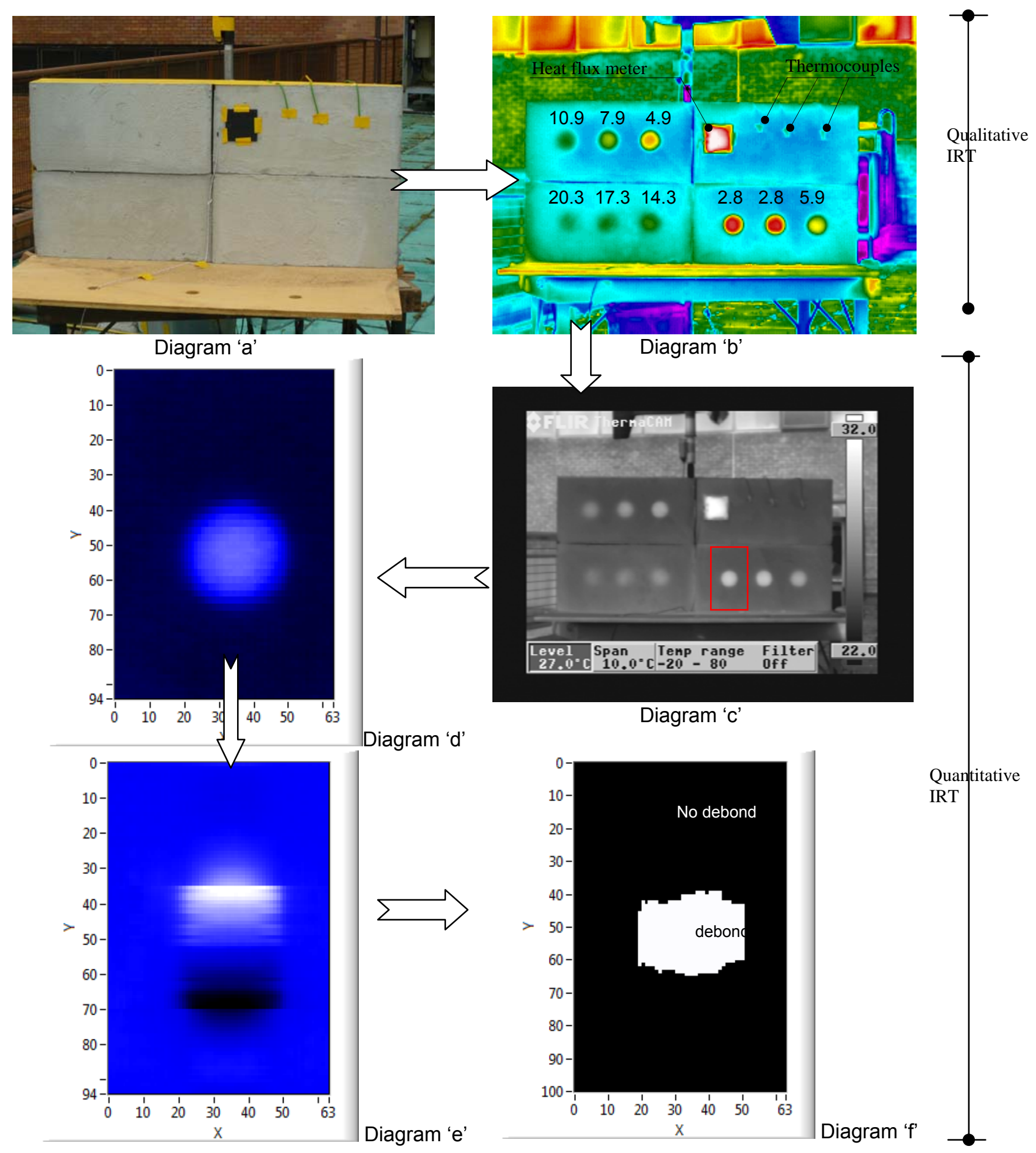

Diagram 'a': Photo taken by camera

Diagram ' $b$ ': Color scale thermogram taken by an infrared thermo-imager. The numbers in the thermogram at the right mean the cover depth of debond.

Diagram 'c': 8-bit greyscale thermogram converted from color scale.

Diagram 'd': Extraction of region of interest (ROI) in red color.

Diagram 'e': Pixel differentiation of the ROI.

Diagram ' $f$ ': Thermogram in binary scale after pixel differentiation.

Fig.2 Methodology of Quantitative Infrared Thermography (QIRT) 
In this paper, a pixel differentiation algorithm based on a second order fit method [1] was adopted. Within the selected region of interest (i.e. red rectangle in Fig. 2, diagram 'c'), the 8-bit pixel intensity P(y) (Fig. 2, diagram 'd') in each column along the vertical direction of the debond was extracted and iterated. Each point in these $P(y)$ was differentiated with respect to the vertical direction ' $y$ ' to determine the maximum gradient which is defined as the position of the debond boundary, at y', as shown in Fig. 2, diagram 'e' and computed using Eq [1].

$$
\frac{\partial P(y)}{\partial y}=0 \text { at } y^{\prime}
$$

where $y=$ pixel cell; and $P(y)=$ pixel intensity as a function of pixel cell.

With the position $y$ ' at every vertical $\mathrm{P}(\mathrm{y})$, the pixel positions outside $\mathrm{y}$ ' was defined as 'intact' and those within ' $\mathrm{y}$ ' was defined as 'debond'. These intact and debond locations over the surface were determined in each vertical section, and the data collected was stacked, and transformed to a binary scale to reveal the distribution of intact and debond areas (Figure 2, diagram ' $f$ '). Finally, the number of pixels defined as debond was then counted and used for the computation of the actual debond area after calculation of the ratio between the number of pixels in the image and the actual width of the panels.

\section{Results and Discussion}

In the experiment on the rendered panels, the heat transfer mechanism was largely governed by the solar heat absorption in the morning, because the panels were placed to face east. The embedded plastic foam plates simulated the debonded areas. They also acted as insulation layers so that the incident heat energy was prevented from dissipation and the surface with the debonds appeared to be hotter than the other intact areas. The most important factor that controlled the intensities of heat absorption was the embedded depth of the plastic foam plates. The closer the plate to the surface is (e.g. $2.8 \mathrm{~mm}$ compared to $20.3 \mathrm{~mm}$ in Figure 2, diagram 'b'), the more obvious and stronger the intensity would be. In other words, the energy increase was dependent on the depth of the foam plates, and therefore it allowed the estimation of the depth of the debonds. The mathematical inversion method of this energy increase has been discussed in Section 3. Two cases with varying heat flux and temperature ranges are reported here, and they are named as (i) hot day and (ii) cold day. Hot day means that the heat flux was larger and the surface temperature was significantly higher than the ambient temperature, and vice versa in the case for the cold day. The larger heat flux and higher temperature collectively infer a larger temperature contrast across the debond and intact surfaces, and illustrate a better visibility for qualitative detection of the debond, as shown by the comparison in Fig. 3. In the hot day, all debonds were easily recognized, while in the cold day, debonds deeper than $10.9 \mathrm{~mm}$ was barely recognizable, despite a smaller temperature range $\left(20-26^{\circ} \mathrm{C}\right)$ was used compared to that in the hot day $\left(22-32^{\circ} \mathrm{C}\right)$. The per-minute changes of heat flux and temperature differential between the render surface and the ambient environment are reported in Figs. 4 and 5 respectively. For both cases, the heat flux and temperature increased steadily when the time approached noon.
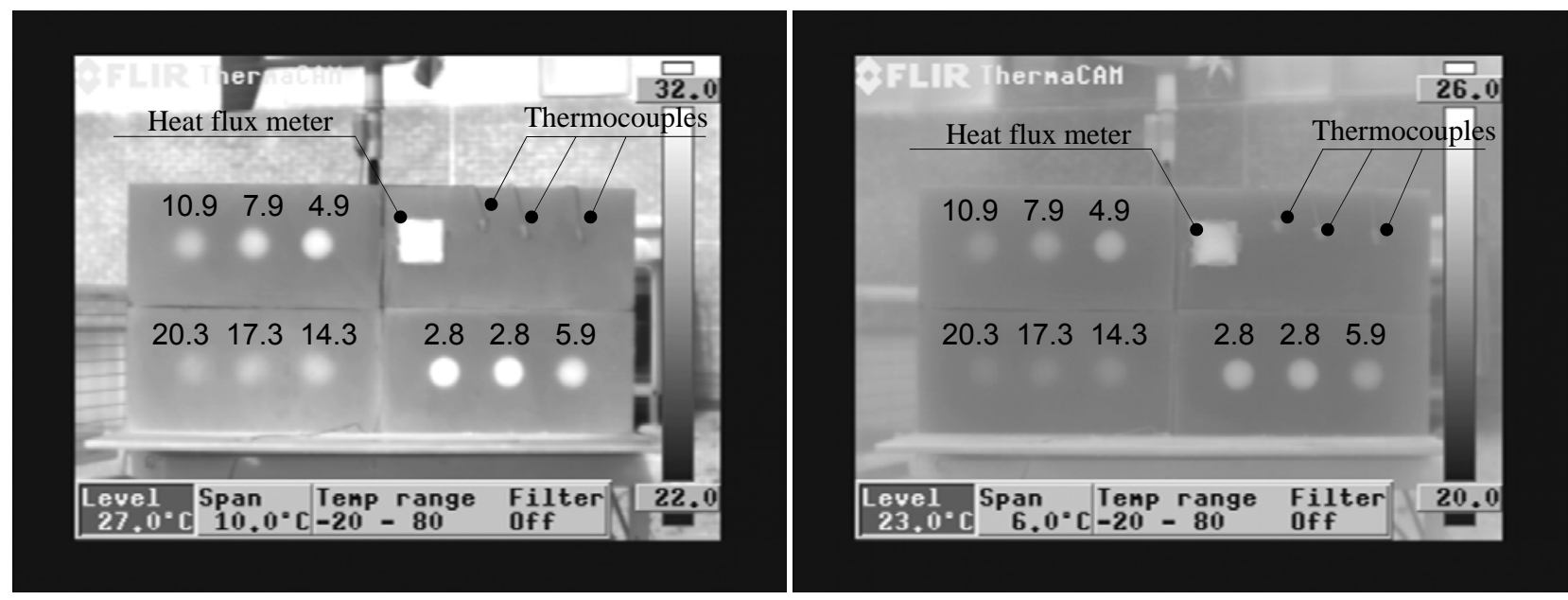

* The numbers in the thermogram at the right mean the cover depth of debond.

Fig. 3 Thermograms captured at 10am in hot (left) and cold day 
From the quantitative perspective, variations in heat flux and temperature do not affect the size determination of the debonds (Figs. 6 and 7) based on the methodology discussed in Section 3. In Figs. 6 and 7, the horizontal black line is the known size of the plastic foam plates which is equal to $\pi r^{2}=44.2 \mathrm{~cm}^{2}(r=3.75 \mathrm{~cm})$. The estimated values of the size of the debond scatter about the black line over the time of IRT measurement. This result suggests that the quantitative inversion method is not affected by the increase of heat flux and temperature differentials, nor by their absolute values. An exception is the under-estimated values of the debond areas located beyond the depth of $17.3 \mathrm{~mm}$ in the case of hot day. However, this underestimation is believed to be caused by the larger disturbance of lateral heat flow for the deeper debonds, but not due to the increase of heat flux.

The root mean squares and the percentage errors of the estimated debond areas are summarized in Table 1. These values are compared to the known values. The results are very encouraging, showing that the percentage errors are at most $<16 \%$ in all cases, which credits to the reliability of the method and algorithm.

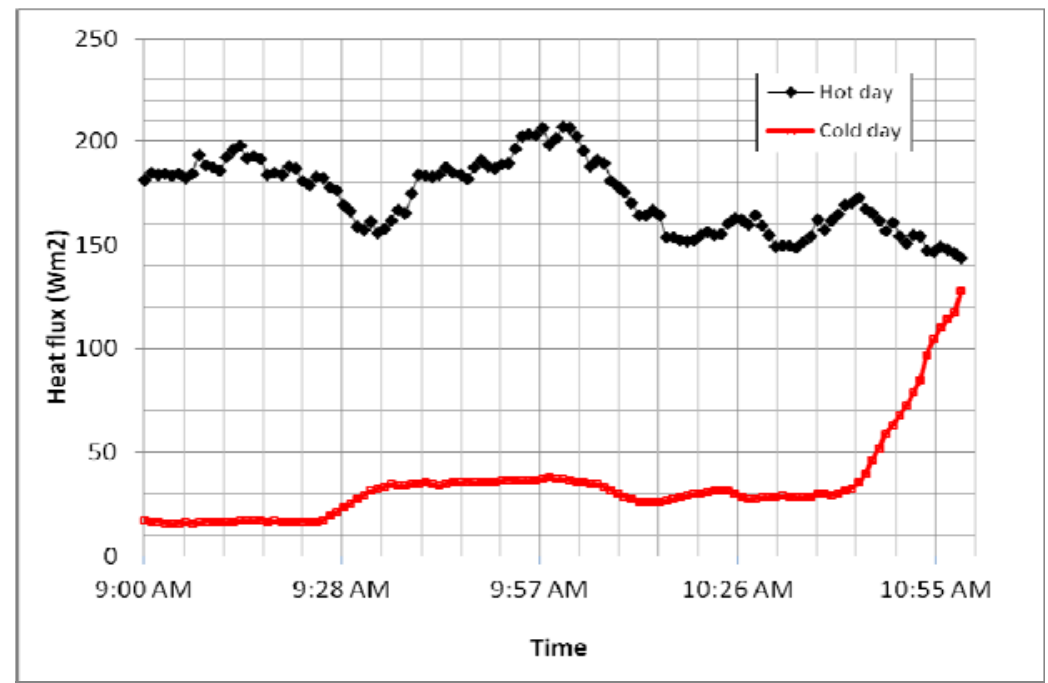

Fig. 4 Change of heat flux over the measurement period

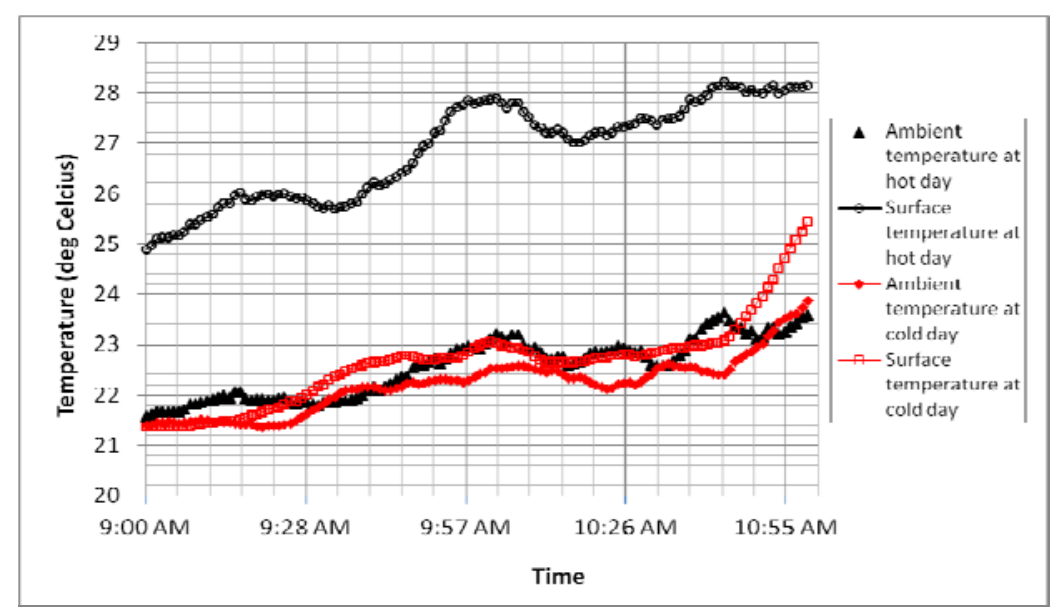

Fig. 5 Change of ambient and surface temperature of the specimens over the measurement period 


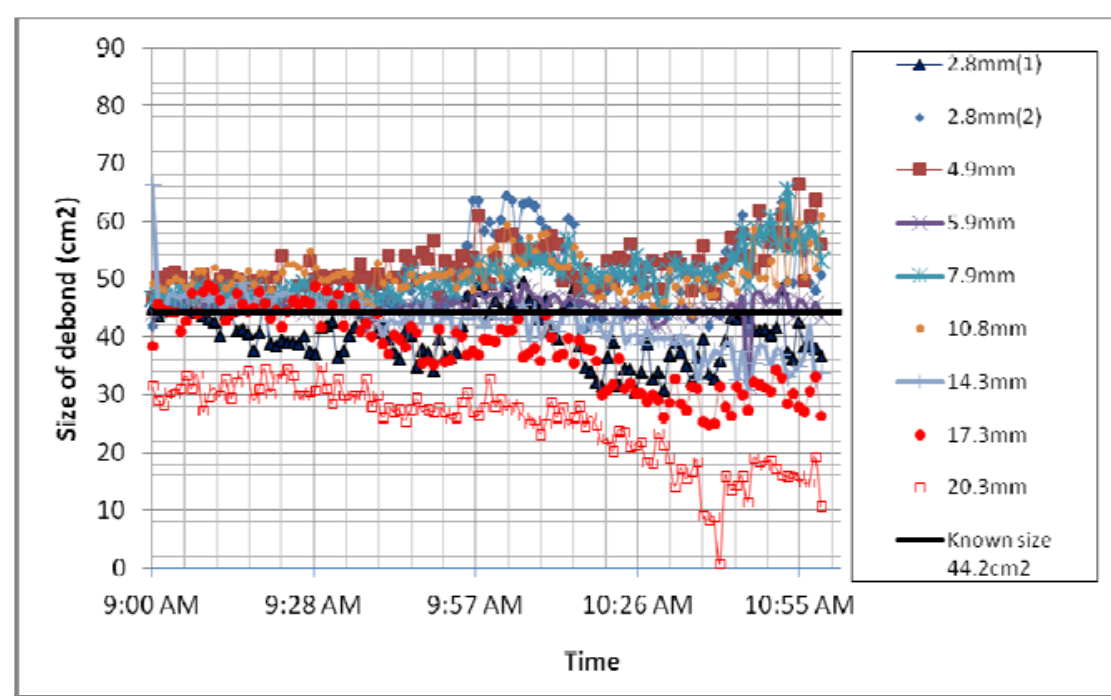

Fig. 6 Change of measured sizes of debond at different embedded depths of the specimens over the measurement period in the hot day

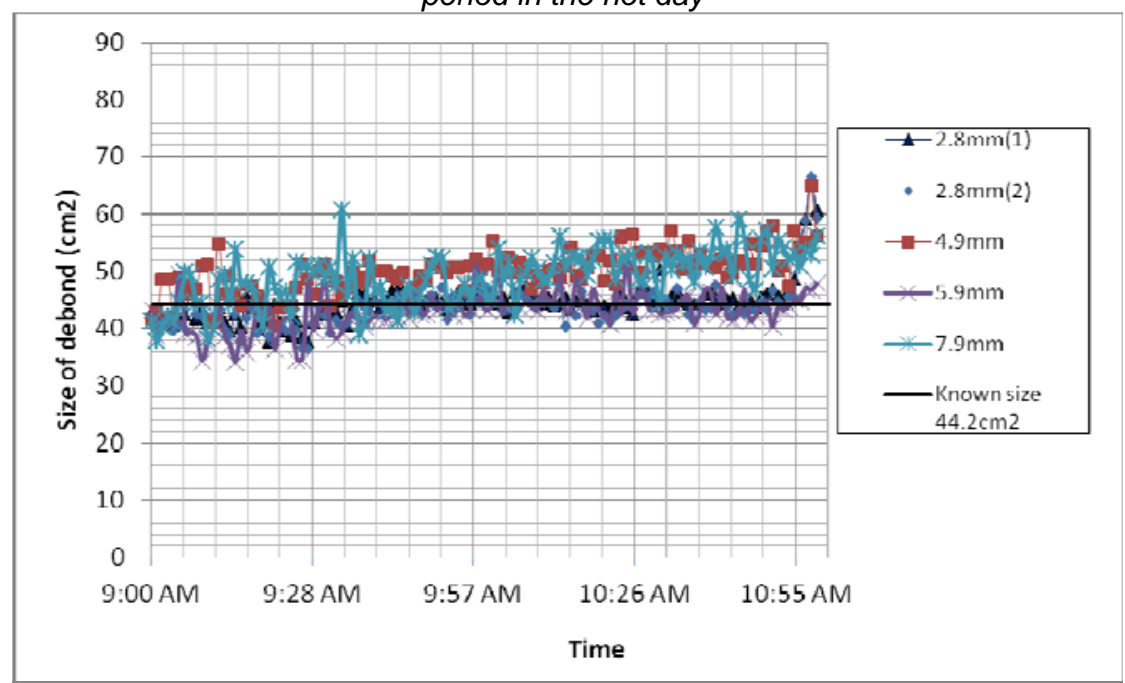

Fig. 7 Change of measured sizes of debond at different embedded depths of the specimens over the measurement period in the cold day

Table 1 Actual and estimested sizes of debonds embedded at various depths

\begin{tabular}{|c|c|c|c|c|}
\hline \multirow{2}{*}{ Size of debond } & \multicolumn{2}{|c|}{ Hot day } & \multicolumn{2}{|c|}{ Cold day } \\
\cline { 2 - 5 } & RMSD (cm2) & \% error & RMSD (cm2) & 8.4 \\
\hline $2.8 \mathrm{~mm}(1)$ & 4.56 & 10.3 & 3.87 & 8.7 \\
\hline $2.8 \mathrm{~mm}(2)$ & 6.33 & 14.3 & 3.85 & 8.7 \\
\hline $4.9 \mathrm{~mm}$ & 3.83 & 8.7 & 3.33 & 7.5 \\
\hline $5.9 \mathrm{~mm}$ & 1.90 & 4.3 & 4.65 & 10.5 \\
\hline $7.9 \mathrm{~mm}$ & 3.72 & 7.6 & Not measurable & Not measurable \\
\hline $10.8 \mathrm{~mm}$ & 3.36 & 10.4 & Not measurable & Not measurable \\
\hline $14.3 \mathrm{~mm}$ & 4.58 & 15.2 & Not measurable & Not measurable \\
\hline $17.3 \mathrm{~mm}$ & 6.72 & 15.4 & Not measurable & Not measurable \\
\hline $20.3 \mathrm{~mm}$ & 6.82 & & & \\
\hline
\end{tabular}




\section{Conclusion}

The method discussed in this paper provides both qualitative and quantitative assessments of debonds in rendered wall finishes. The method is based on passive infrared thermography when it is subjected to changes of heat flow and the solar intensity. The results show that the error of debond size estimation is within $16 \%$ which is considered to be good in non-destructive evaluation. Other factors affecting the results (e.g. angle of illumination, wind speed, variation of emissivity, types of thermo-imagers, etc.) are being investigated with the view to further improve the accuracy of prediction.

\section{Acknowledgement}

The authors wish to thank the funding support of the Innovative Technology Fund, HKSAR Government, and Optimix Cement Connections Ltd. for providing the plastering materials.

\section{REFERENCES}

[1] Maldague, X. P. V. Theory and Practice of Infrared Technology for Nondestructive Testing, Wiley-Interscience, John Wiley \& Sons, Inc. 2001.

[2] ACl 2282r-98, Nondestructive Test Methods for Evaluation of Concrete in Structures

[3] BS EN 13187:1998 Thermal performance of buildings - Qualitative detection of thermal irregularities in building envelopes - Infrared method

[4] Hong Kong Concrete Institute, Test Method for Evaluation of Building Exterior Wall Finishes using Infrared ThermalImaging Technique, 2009.

[5] W.L. Lai, S. C. Kou, C.S. Poon, W.F. Tsang, S.P. Ng and Y.Y. Hung. Characterization of flaws embedded in externally bonded CFRP on concrete beams by infrared thermography and shearography, Journal of Nondestructive Evaluation, Volume 28, pp. 27-35, 2009.

[6] W.L. Lai, S. C. Kou, C.S. Poon, W.F. Tsang, C. C. Lai. Effects of elevated temperatures on interfacial delaminations, failure modes and shear strength in externally-bonded CFRP beams using infrared thermography, gray-scale images and direct shear test, Construction and Building Materials, Volume 23, pp. 3152-3160, 2009.

[7] W. L. Lai, S. C. Kou, C. S. Poon, W. F. Tsang, C. C. Lai. Characterization of the Deterioration of Externally Bonded CFRP-Concrete Composites using Quantitative Infrared Thermography, Cement and Concrete Composites, 32(9): 740746, 2010.

[8] Optimix, BP126 base plaster product catalogue.

[9] W.L. Lai, S. C. Kou, Poon C.S., Tsang W.F., Ng S.P. and Hung Y.Y. Characterization of flaws embedded in externally bonded CFRP on concrete beams by infrared thermography and shearography, Journal of Nondestructive Evaluation, 28: 27-35, 2009.

[10] Krapez, J.C. and Cielo, P. Thermographic nondestructive evaluation: data inversion procedures part I: 1D analysis, Res. Nondestructive Evaluation, 1991, 3(2), 81-100.

[11] Gonzalez, R.C. and Wintz, P. Digital Image Processing, 2nd Ed. Addision-Wesley, Reading, MA, 1987.

[12] Starnes, M.A., Carino, N.J., Kausel, E.A. Preliminary Thermography Studies for Quality Control of Concrete Structures Strengthened with Fiber-Reinforced Polymer Composites. Journal of Materials in Civil Engineering, Volume 15, No. 3, pp. 266-273, 2003. 